\title{
A REVIEW OF EXPECTED CHANGES TO CONTINUING PROFESSIONAL DEVELOPMENT (CPD)
}

\author{
A Craven, Associate Advisor in General Practice \\ Meadowside Surgery, Lancaster
}

\section{KEY FEATURES}

- all NHS healthcare workers will be required to produce a personal development plan by April 2000

- all GPs will be required to produce a personal and practice development plan by April 2000

- although PGEA will overlap with the new system, it is likely to be completely replaced within two years

\section{INTRODUCTION}

The information in this article is of most use to GPs but hopefully also to hospital staff. This article will give a very personal view on the current state of play in GP education and how it will effect us all. I am acutely aware that we must maintain our professional and social contacts with colleagues and that these seem to be threatened by Primary Care Group (PCG) and educational developments.

In 1998 the Chief Medical Officer (CMO) produced a review of continuing professional development (CPD) in general practice. Although this was distributed to all GP practices, the implications of this document are not widely understood.

During a presentation at a recent national conference it was stated by the NHSE that all health workers in the NHS will need a personal development plan by April 2000. Nurses and some other paramedical staff are well ahead of the medical staff as they already work with portfolios. This brief article will summarise the key points in the CMO review, then outline how the changes may effect the primary healthcare team, other attached practice staff and hospital staff.

\section{DEFINITION OF CPD}

Members of the CPD review group gave the following definition: "A process of lifelong learning for all, to meet the needs of the patients and deliver the priorities of the NHS".

\section{SUMMARY OF THE CPD REVIEW}

Why a review?

1. General practice works best through a multidisciplinary approach. Under the current arrangements there is little opportunity for cross-professional learning before and after qualification.

2. There is a lack of coherent planning or provision of educational opportunities, although good examples exist.
3. The PGEA system (limited to GPs) fails to demonstrate any convincing benefits for patient care and is often not needs-based.

CPD related to GP practice should:

- Be purposeful and personally motivating

- Raise individual awareness of learning needs

- Consider the development needs of the practice

- Be evidence-based where possible

- Develop knowledge of the opportunities for research and development

- Place the individual at the centre of the educational process

- Take account of uni-professional and multi-professional learning needs.

Any new system should encourage practice and personal development, and use a multidisciplinary approach to working and learning.

\section{THE WAY FORWARD}

The principal proposal of the CPD review is to integrate and improve the educational process through a practice professional development plan.

This would look at the development needs of the practice as a whole and for each health professional within it. It will develop the concept of the "whole practice" as a resource for healthcare.

\section{THE FUTURE OF PGEA}

The CPD review states that PGEA will be replaced by personal development plans. The NHSE has stated that each health worker should have a personal educational development plan by April 2000. This will be integrated into a practice development plan.

The CPD review states that there will be an overlap between the two systems, but it is likely that PGEA will be replaced within two years. At the present time it is not clear how the system will work although it is hoped that a working group will report this autumn. In the meantime, practices are presenting development plans for PGEA approval via the GP tutors. 


\section{THE PRACTICE DEVELOPMENT PLAN AND PRACTICE-BASED LEARNING}

There is no official guidance on producing either personal or practice development plans. It is felt that the plan should be needs-based and not all wants- and interest-based, easy to follow through, specific, and should contain methods of evaluation. The plan should not be totally driven by the clinical governance or Health Authority agenda.

Several practices in the northwest have been trying to develop basic education plans based on perceived need. This has been done with all members of the primary healthcare team asking the questions: what skills do we have in the practice? what skills do we need in the practice? how can we best achieve this? This has successfully enabled the production of basic plans which can be reviewed and followed up. As an extension to this, these have been used with hospital SHOs to try to encourage them to look at what they need from a six-month hospital post.

These ideas have now been refined to take into account practice, local/PCG and national priorities (Figures 1 and 2). If these are taken on by individual practices it is likely to fulfill all the requirements for CPD and be an essential part of reaccreditation.

\begin{tabular}{|l|}
\hline NAME: \\
\hline My own strengths in my job are: \\
\hline My own weaknesses in my job are: \\
\hline $\begin{array}{l}\text { Over the last twelve months, the following have changed my } \\
\text { practice: }\end{array}$ \\
\hline Over the last twelve months, I have become better at: \\
\hline $\begin{array}{l}\text { Over the past twelve months, I have been particularly helped by: } \\
\text { (any training, reading or meetings) }\end{array}$ \\
\hline Over the past twelve months, I have been particularly hindered by: \\
\hline I have used the following methods to identify my training needs: \\
\hline Over the next twelve months I plan to: \\
\hline I will achieve this by: (methods of education) \\
\hline I will be able to evaluate my learning by: \\
\hline In the longer term, I would like to: \\
\hline My learning will benefit the practice by: \\
\hline
\end{tabular}

Figure 1 Personal learning plan

(adapted from Stephen Holmes, GP tutor)

There is some concern that educational meetings will be pulled out of post graduate centres. Although this may occur it does not have to be the case and would be very inward-looking.

Future postgraduate events could include, for example:

- Practice-specific events

- Specific courses

- Audit between practices and the MAAG

\section{PLEASE ANSWER THE FOLLOWING QUESTIONS FOR THE PRACTICE AS A WHOLE TEAM}

What are the learning needs of the practice?

How have these been identified: (discussions, surveys, audit, significant event analysis)

How do these reflect priorities in the wider NHS, within the health authority and the PCG?

How will the learning needs be met?

How will the success of the learning be evaluated over the next year?

Figure 2 Practice professional development plan

(adapted from Stephen Holmes, GP tutor)

- Significant event analysis

- Workshops at the practice or hospital

- Lectures

The GP tutor or PCG may identify common needs across practices and decide to put on a workshop or meeting at the postgraduate centre in conjunction with the clinical tutor, who will be aware of hospital priorities. Different levels are, therefore, required. One example of the kind of issue which may arise is mental health.

- This is a national priority and a national framework is being produced. It will therefore be on the HA and PCG agenda. The education and training consortia also see it as a priority. The HA and PCG could apply to the education and training consortia for funding of educational events. Workshops can be organised by the GP and clinical tutors for the multidisciplinary team and hospital staff.

- The practice may feel it is a priority and carry out an audit on an aspect of mental health. This could be followed up by a meeting of the primary healthcare team, with CPN or consultant input if needed.

- One of the partners may be section 12 approved and need to attend a specific update course on the Mental Health Act.

All of these can be part of a personal or practice development plan, and these ideas can easily be adapted for hospital staff.

\section{EVALUATION OF EDUCATIONAL EVENTS}

It is often difficult to demonstrate that an event has been effective or that the participants have learnt anything. There will need to be some write-up of an event and some attempt to evaluate it. This may range from a simple evaluation form to an audit demonstrating change or the production of a protocol.

\section{MULTIDISCIPLINARY WORKING AND EDUCATION}

This was the second key principal of the CPD review. Headrick et $a l^{(2)}$ discuss this subject and recognise some barriers to interprofessional working and education 
Differences in history and culture

Historical interprofessional and intraprofessional rivalries

Differences in language and jargon

Differences in schedules and professional routines

Varying levels of preparation, qualification and status

Differences in requirements, regulations and norms of professional education

Fears of diluted professional identity

Differences in accountability, payment and rewards

Concerns regarding clinical responsibility

Figure 3 Barriers to interprofessional collaboration and education

(Figure 3). Most health professionals, however, have one characteristic in common, a desire to learn; and at least one shared value, to meet the needs of their patients. Further, adults learn best when the topic in hand is close to their interests. A powerful incentive to teamwork is created by directing attention to areas where changes are likely to result in measurable improvements for the patients.

It has been said that the more time people spend together the more they understand each other and the better they work together. Significant event analysis ${ }^{(3)}$ lends itself especially to this type of education and is extremely effective in sorting out complex problems within a practice, improving working conditions and patient care.

\section{CONCLUDING COMMENTS}

I have tried to summarise what I feel are the key areas effecting CPD. These changes are coming and will need to be taken on board. I am sure that all the agendas of education, reaccreditation and clinical governance are linked and should be dealt with together. This will all take time and it should be noted that in other areas practices are encouraged to have protected time similar to the monthly hospital meetings with the cooperatives covering for emergencies.

It is essential that the educational leads of the PCGs, GP tutors, clinical tutors, nurse tutors, university postgraduate departments, MAAG and others involved with education and clinical governance are working together and are moving in the same direction.

\section{REFERENCES}

1 A Review of Continuing Professional Development In General Practice. Department of Health. May 1998

2 Headrick LA, Wilcock PM, Batalden PB. Interprofessional working and continuing medical education $\mathrm{Br}$ Med J 1998;316:771-4

3 Significant Event Auditing RCGP Occasional Paper. March 1995

\section{Acknowledgments}

Figures 1 and 2 are from Dr S Holmes, GP tutor in Burnley. Figure 4 is from Professor Patrick Pietroni and Anne Kilcoyne.

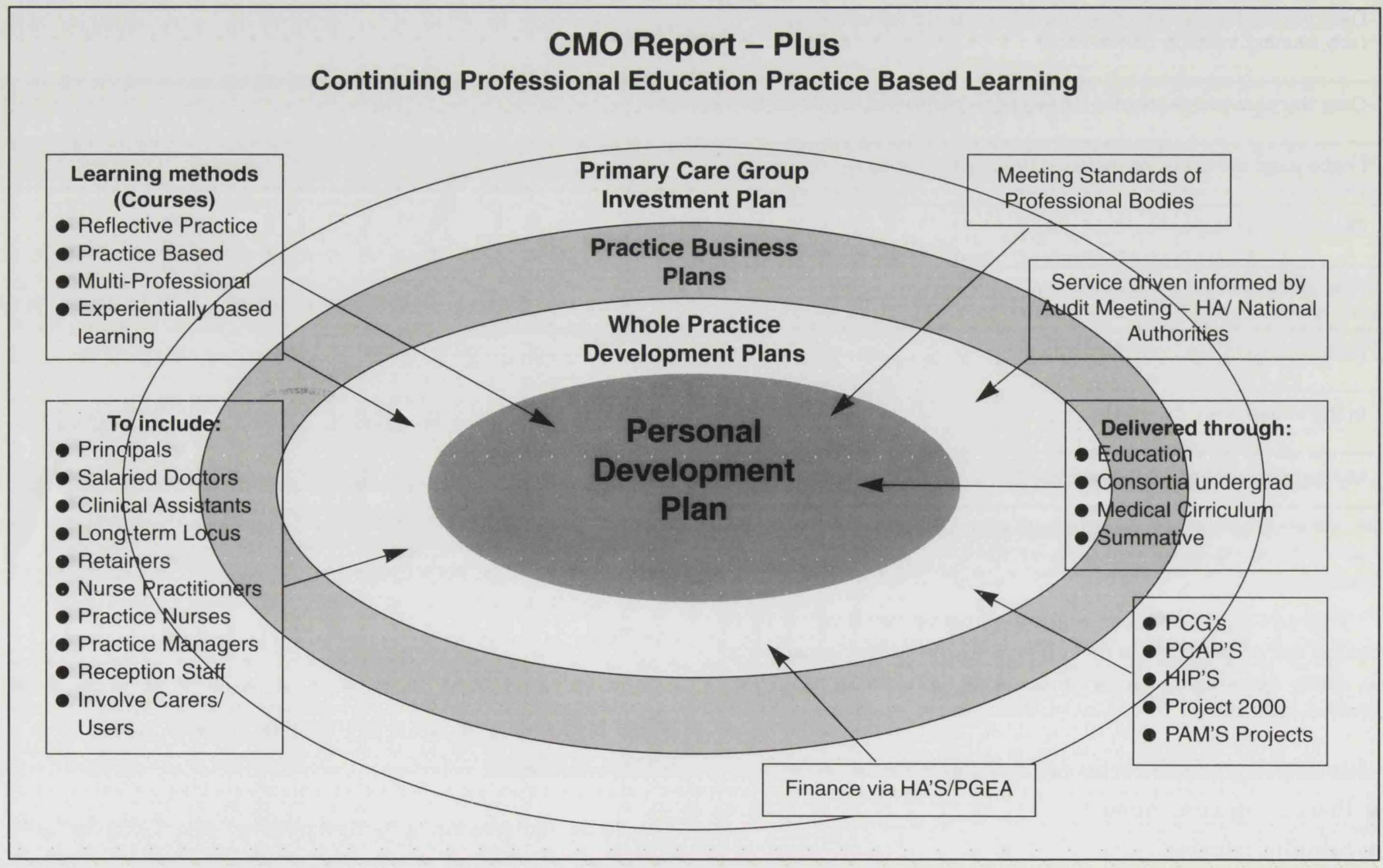

Figure 4 Patrick Pietroni's diagram - How it could look 